\title{
One-parameter modified nonrandom two-liquid (NRTL) activity coefficient model
}

\author{
Solomon Gebreyohannes ${ }^{1}$ \\ Brian J. Neely ${ }^{1}$ \\ Khaled A. M. Gasem ${ }^{1,2 *}$
}
'Oklahoma State University School of Chemical Engineering Stillwater, OK 74078
${ }^{2}$ University of Wyoming
Department of Chemical \& Petroleum Engineering Laramie, WY 82071

* email: gasem@uwyo.edu

Phone: (307) 766-2973

Fax: (307) 766-2606 


\section{Abstract}

The nonrandom two-liquid (NRTL) model is utilized widely in phase equilibria calculations and employs three adjustable parameters (two interaction parameters and the non-randomness factor) that are determined through regression of experimental data for a specific binary vaporliquid equilibrium (VLE) system. The two interaction parameters ( $a_{12}$ and $a_{21}$ ) account for the difference between the pure-component liquid interactions $\left(g_{11}\right.$ and $\left.g_{22}\right)$ and mixed-component liquid interactions $\left(g_{12}\right.$ and $\left.g_{21}\right)$. The main disadvantage of the NRTL model is the strong correlation between the two parameters of the model.

In this work, we propose a new modification to the NRTL model which addresses the limitation of the original model. The newly modified model recasts the formulation of the NRTL model parameters in such a way that the new parameters reflect two different characteristics. It also allows one of the two new model parameters to be generalized in terms of pure-component properties, which reduces the NRTL model to a one-parameter model. The original and modified NRTL models yield comparable precision in representing experimental equilibrium properties. Some of the benefits of our modification include easy generalizability of the parameters, ability to classify VLE behaviors based on a single model parameter and fewer convergence problems in parameter regressions.

Keywords: NRTL, activity coefficient, one parameter NRTL model, phase behavior 


\section{Introduction}

Phase equilibrium properties, such as pressure, temperature, compositions and partition coefficients are required for the design of chemical separation operations. Generalized thermodynamic models are used widely to describe phase equilibria properties of systems; thus avoiding the need to conduct expensive and time intensive experimental property measurements.

In phase equilibria calculations, activity coefficients $(\gamma)$ are used to account for component nonideal liquid behavior in a mixture. A number of activity coefficient models for representing vaporliquid equilibrium (VLE) and liquid-liquid equilibrium (LLE) systems have been proposed by various researchers [1-5]. These models demonstrate the composition and temperature dependence of activity coefficients. In general, the literature models can be classified as historical semi-empirical activity coefficient models (Margules [6], Redlich-Kister [6] and Van Laar [6]), theory-based models, which include local composition and two-liquid models (Wilson [7], NRTL [1] and UNIQUAC [3]) and group-contribution models (UNIFAC [2], ASOG [8]).

The NRTL model is among the most widely used activity coefficient models in phase equilibria. The model requires three adjustable parameters, which include two energy interaction parameters $\left(a_{12}\right.$ and $\left.a_{21}\right)$ and a non-randomness factor $\left(\alpha_{12}\right)$. The model provides good representation of experimental equilibrium data for strongly non-ideal mixtures and partially immiscible systems [6].

One of the main disadvantages of the NRTL model is the strong correlation between the two energy interaction parameters $\left(a_{12}\right.$ and $\left.a_{21}\right)$. A number of researchers [9-13] have attempted to modify the original NRTL model to eliminate or reduce the correlation. Many of the modified models presented in the literature, however, were not successful in decoupling the energy interaction parameters between like and unlike molecules. Further, they lacked simplicity and 
were not evaluated for wide range of interactions. Therefore, a need exists for a simple modification of the NRTL model that eliminates the parameter correlation.

In this work, we propose a new modification to the NRTL activity coefficient model addressing the limitation of the original model. The newly modified model recasts the model parameters in such a way that the two new model parameters reflect two different characteristics, namely energy interaction and energy interaction ratio parameters. This new modification enables easier generalization of one of the parameters (energy interaction ratio) in terms of purecomponent properties, which essentially reduces the NRTL model to a one-parameter model for a VLE system. As such, our modification eliminates the parameter correlation present in the original model by reducing the number of model parameters. The single model parameter also provides a capability of relating/classifying VLE behaviors based on that parameter value. The ability to identify behaviors of systems with only the parameter value is useful for designing processes involving new systems.

The objectives of this work are (1) to mitigate the limitations of the original NRTL model, namely the parameter correlation and generalizability, (2) to evaluate the representation capability of the original and modified NRTL models for representing mixtures containing various functional groups, (3) to provide a qualitative approach to classifying systems in terms of their behaviors, (4) to assess the applicability of the original and modified NRTL models for multicomponent systems and (5) to evaluate the temperature dependence of model parameters.

To meet the objectives of this work, VLE and LLE databases were assembled from the DECHEMA-VLE [10], DECHEMA-LLE [14], NIST-TDE [15] and DIPPR [16] databases. The VLE systems were classified by chemical class and a qualitative approach was applied to classify the systems by behavior into nearly-ideal, non-ideal and highly non-ideal systems. Further, the data were used to validate the applicability of the models for multiphase and "cross-phase" property predictions, i.e., the applicability of LLE regressed parameters to predict VLE properties and vice versa. 


\section{Literature review on one-parameter NRTL activity coefficient models}

The nonrandom two-liquid (NRTL) activity coefficient model developed by Renon and Prausnitz in 1968 [6] is based on the local composition theory of Wilson [7] and the two-liquid solution theory of Scott [17]. The model provides precise representation of highly non-ideal VLE and LLE systems. The NRTL activity coefficient expression for components in a binary system is given as:

$$
\ln \gamma_{i}=x_{j}^{2}\left[\tau_{j i}\left(\frac{G_{j i}}{x_{i}+x_{j} G_{j i}}\right)^{2}+\frac{\tau_{i j} G_{i j}}{\left(x_{j}+x_{i} G_{i j}\right)^{2}}\right]
$$

where $\tau_{i j}$ and $G_{i j}$ are defined as:

$$
G_{i j}=\exp \left(-\alpha_{i j} \tau_{i j}\right) \quad \tau_{i j}=\frac{g_{i j}-g_{i j}}{R T}=\frac{a_{i j}}{R T}
$$

where $\alpha_{i j}$ is the non-randomness factor in the mixture, $g_{i j}$ is energy interaction between $i$ and $j$ component molecules, $a_{i j}$ is energy interaction difference of $g_{i j}$ and $g_{j j}, x_{i}$ is the mole fraction of component $i, R$ is the universal gas constant and $T$ is the mixture temperature.

The NRTL model contains three parameters (defining $\alpha_{i j}=\alpha_{j i}$ ) that are specific for each binary system. These adjustable parameters are $a_{12}$ or $\left(g_{12}-g_{22}\right), a_{21}$ or $\left(g_{21}-g_{11}\right)$, and $a_{12}$. The two energy interaction parameters account simultaneously for pure-component liquid interactions $\left(g_{11}\right.$ and $\left.g_{22}\right)$ and mixed-liquid interactions $\left(g_{12}\right.$ and $\left.g_{21}\right)$. The non-randomness factor $\left(\alpha_{12}\right)$ varies from 0.2 to 0.47 [6] and can often be set a priori. To be consistent with the DECHEMA database [14] and to accommodate liquid-liquid equilibrium systems, the nonrandomness factor was kept constant as 0.2 for all binary systems in this work. 
As mentioned previously, the parameters $a_{12}$ and $a_{21}$ in the basic NRTL model are strongly correlated. A number of researchers have tried to modify the NRTL model to eliminate or reduce the correlation between the parameters. Bruin and Prausnitz in 1971 [9] first attempted to reduce the number of parameters in the NRTL model. They presented a new derivation of the model by substituting the local mole fractions with volume fractions. Their newly derived equation is shown, as follows, in Equation 3:

$$
\begin{aligned}
& \ln \gamma_{i}=q_{i j} \Phi_{j i}{ }^{2} \tau_{j i}+q_{j i} \frac{x_{2}}{x_{1}} \Phi_{i j}\left(1-\Phi_{i j}\right) \tau_{i j} \\
& \Phi_{i j}=\frac{v_{i} x_{i} \exp \left(-\alpha_{i j} \tau_{i j} / q_{j i}\right)}{v_{j} x_{j}+v_{i} x_{i} \exp \left(-\alpha_{i j} \tau_{i j} / q_{i j}\right)} \\
& \tau_{i j}=\frac{g_{i j}-g_{j j}}{R T}
\end{aligned}
$$

where $\Phi_{i j}$ is the local volume fraction of molecule $i$ around molecule $j ; v_{j}$ is the molar volume of molecule $i ; q_{i j}$ is a measure of the number of sites a molecule of type $i$ occupies in a pseudo lattice structure.

The newly introduced $q_{12}$ and $q_{21}$ parameters in the modified equation are determined using the following three conditional statements:

$$
\begin{array}{lll}
v_{1}>v_{2} & q_{12}=\left(\frac{v_{1}}{v_{2}}\right)^{1 / 2} & q_{21}=1 \\
v_{1} \approx v_{2} & q_{12}=1 & q_{21}=1 \\
v_{1}<v_{2} & q_{12}=1 & q_{21}=\left(\frac{v_{2}}{v_{1}}\right)^{1 / 2}
\end{array}
$$

For further simplification of the model, Bruin and Prausnitz [9] suggested the pure energy interaction parameters $\left(g_{11}\right.$ and $g_{22}$ ) be estimated from pure component property data specifically using the internal energy of complete vaporization, which is shown as follows in Equation 5: 


$$
g_{i i}=-\beta E_{i}
$$

where $\beta$ is the proportionality constant and $E_{i}$ is the energy change upon isothermal vaporization from the saturated liquid $i$ to the ideal-gas state. An expression for $E_{i}$ can be derived from the Clausius-Clapeyron equation [9]. Such modification leaves $g_{i j}$ as the only adjustable parameter in Equation 3.

Bruin and Prausnitz [9] tested six variations of the modified NRTL model using 130 binary VLE systems in which about 50 of the systems were aqueous systems. Two of these variations were; (1) NRTL with equal molar volumes $\left(v_{1}=v_{2}\right)$ and one adjustable parameter and (2) NRTL with different molar volume, size factor (q) and one adjustable parameter. When the first model was considered, the average \%AAD (average absolute percentage deviation) in pressure and AAD (average absolute deviation) in vapor mole fraction were approximately 2 to 8 times the error found using the original NRTL model for aqueous systems. Their second model resulted in comparable errors in pressure and vapor mole fraction to that of the original NRTL model. Although the error reduced significantly in the second case, the equation has an added complexity and conditional statements, as shown in Equation 4.

Vetere in 1977 [18] followed the work of Bruin and Prausnitz [9] on generalizing the parameters of the NRTL model. He proposed an empirical method to estimate the parameter $g_{i j}$ in the NRTL model. The proposed model employs a modified form of Equation 5 to determine the two pure interaction NRTL parameters $g_{11}$ and $g_{22}$, as shown in Equation 6:

$$
\begin{aligned}
& g_{i i}=-\left(\Delta H_{v i}-R T\right) \\
& g_{i j}=f\left(\delta_{i}, \delta_{j}\right)
\end{aligned}
$$

where $H_{v}$ is heat of vaporization and $\delta$ is the Hildebrand solubility parameter of a pure compound. 
The only unknown parameter $g_{i j}$ is determined by using the Hildebrand solubility parameters of the pure compounds. Vetere [11-13] showed the use of various empirical forms of the above concept to estimate the cross-interaction parameter $\left(g_{i j}\right)$ of the NRTL model. For aqueous and non-aqueous systems, he presented the following equations to determine $g_{i j}[12,13]$ :

$$
\begin{aligned}
& \left(g_{i j}-g_{i i}\right)+\left(g_{i j}-g_{j j}\right)=A+B\left(\delta_{i}-\delta_{j}\right) \\
& \frac{\tau_{j i}}{\tau_{i j}}=a+b\left(g_{i i}-g_{j j}\right)
\end{aligned}
$$

The values of the new parameters $a, b, A$ and $B$ are generalized for five aqueous and five nonaqueous classes of mixtures. All four parameters were regressed for each chemical class using selected binary systems. The modified NRTL model was evaluated using over 60 binary nonaqueous and a limited number of aqueous systems. The predictive capability of the model was comparable to that of the UNIFAC-1991 [19] model for the selected systems.

Although the generalized model provided an alternative for estimating $g_{i j}$, the model suffers various limitations, which includes a lack of simplicity because of the additional empirical equations and the four parameters which need to be regressed for each type of chemical class. Another limitation is the inability to define effectively the chemical class of a compound in a binary system. Further, the parameter generalization was conducted for a small number of binary systems; therefore, the model had limited capability to predict VLE properties of systems with diverse functional-group interactions.

\section{Modified NRTL models}

In this section, we present the proposed modified two-parameter NRTL (mNRTL2) and modified one-parameter NRTL (mNRTL1) models for VLE and LLE systems. The first model (mNRTL2) recasts the original NRTL model parameters so that the two new parameters reflect different 
characteristics. In the second model (mNRTL1), pure-component properties are used to generalize one of the parameters of the mNRTL2 for VLE systems.

\subsection{Two-parameter modified NRTL model (mNRTL2)}

The NRTL model parameters can be written as follows:

$$
a_{i j}=\left(g_{i j}-g_{j j}\right)=g_{i j}\left(1-\frac{g_{j j}}{g_{i j}}\right)=g_{i j}\left(1-r_{i j}\right)=g_{i j} R_{i j}
$$

Thus, the original two-parameter NRTL model is presented in terms of a binary interaction energy parameter, $g_{i j}$, and interaction ratio, $R_{i j}$, which represents the relative deviation or difference of $g_{i j}$ from $g_{j j}$. Here, various empirical modeling alternatives could be proposed as potential modifications of the NRTL model. The following scenarios were investigated in this study.

1: Estimate the pure-component energy interaction parameters as a function of heat of vaporization of pure components. This was a similar modification to that of Bruin and Prausnitz [9] model.

2: Assume geometric mean of the pure-component energy interactions $\left(g_{j j}\right.$ and $\left.g_{i i}\right)$ to estimate the binary energy interaction parameter $\left(g_{i j}\right)$.

3: Assume conformal combination rule [20] to estimate the binary energy interaction parameter from the pure-component energy interaction parameters.

4: Assume half harmonic mean of the pure-component energy interactions to estimate the binary energy interaction parameter.

Our investigation shows scenario 4 to produces consistently good results. This combined with its simplicity and ease of generalization, motivated us to adopted scenario 4 in our modified 
$g_{i j}$ is expressed as:

$$
g_{i j}=\left[a_{j i} a_{i j}\right]^{1 / 2}=\left[\left(g_{i j}-g_{i i}\right)\left(g_{i j}-g_{j j}\right)\right]^{1 / 2}=\left[\frac{g_{i i} g_{j j}}{g_{i i}+g_{j j}}\right]
$$

Further, substituting Equation 9 into Equation 8, the interaction ratio $\left(\mathbb{R}_{\overline{\mathrm{d}} \bar{j}}\right)$ becomes,

$$
R_{i j}=\frac{1}{R_{j i}}
$$

Thus, we obtain a modified two-parameter NRTL model (mNRTL2) with two adjustable parameters $\left(g_{i j}\right.$ and $\left.R_{i j}\right)$. The modified two-parameter NRTL model works for most of the VLE and LLE systems studied. The effectiveness of the model has been evaluated and compared with the original NRTL model. Results show comparable representations of phase equilibria properties. We also have observed that $g_{i j}$ and $R_{i j}$ are easier to regress than the original NRTL parameters $a_{12}$ and $a_{21}$. The $g_{i j}$ and $R_{i j}$ values range from -500 to 1500 and from 0 to 4 , respectively. Our analysis shows, compared to the original NRTL, the mNRTL2 model is easier to initialize. Values of $g_{i j}=200$ and $R_{i j}=1$ tend to be good initial values for most of the VLE systems. Further, the mNRTL2 model has a slightly lower correlation coefficient value of 0.94 compared to the original NRTL model which resulted in a correlation coefficient value of 0.97 for the VLE systems considered in this study.

\subsection{One-parameter modified NRTL model (mNRTL1)}

A generalization for the interaction ratio $R_{i j}$, in the proposed mNRTL2 model was obtained in terms of pure-fluid properties. After evaluating the ratios of various pure fluid properties, the ratio of acentric factor and critical pressure resulted in the best representation of equilibrium properties of VLE systems, as shown in the following equation: 


$$
R_{i j}=\left[\left(\frac{\omega_{j}}{\omega_{i}}\right)\left(\frac{P_{C i}}{P_{C j}}\right)\right]
$$

where $\omega$ is acentric factor, $P_{c}$ is critical pressure and the $i$ and $j$ subscripts are molecules type $i$ and $j$, respectively. Use of Equation 11 permits $R_{i j}$ to be determined from pure substance properties, leaving only one parameter, $g_{12}$, to be regressed.

Equation 11 shows the ratio of pure-fluid properties in the modified one-parameter NRTL model (mNRTL1) that are used to determine the interaction ratio parameter. This modification essentially reduces the NRTL model to a single parameter model. As discussed below, this modified model is capable of describing VLE proprieties, as well as the infinite limits of the equilibrium properties.

\section{Representation of equilibrium experimental data}

The representation capabilities of the proposed models were evaluated using a comprehensive database of VLE experimental data. The database was assembled from available sources by insuring sufficient representation of a variety of functional groups in the database. The experimental VLE data were taken from DECHEMA [10] and NIST-TDE [15]. The purecomponent vapor pressure data were collected from DIPPR [16] and DECHEMA [10].

\subsection{VLE database}

A low-pressure binary VLE database (Oklahoma State University, OSU database I) consisting of 188 binary VLE systems totaling 4716 data points was assembled [21]. This database is comprised of systems of aliphatic and aromatic hydrocarbons, water, alcohols, ethers, sulphides and nitrile compounds. A second database, comprised of 388 binary VLE systems totaling 12,010 data points, was taken from DECHEMA [10]. A third database consisting of 340 binary systems totaling over 17,000 data points was taken from NIST-TDE [15]. In total, the database compiled in this work consists of a total of 916 binary systems formed from various 
combinations of 140 different compounds. A total of over 33,000 vapor-liquid equilibrium data points were assembled in the final database (Oklahoma State University, OSU-VLE Database III). The data covered a temperature range from 128 to $554 \mathrm{~K}$ and pressures to $58 \mathrm{bar}$; however, over $99 \%$ of the data were at pressure of less than 10 bar. In addition to pressure, temperature and mole fraction (PTXY) data, we have collected over 500 data points of infinite-dilution activity coefficient values $\left(\gamma^{\infty}\right)$ for 137 of the 916 VLE systems in the database [10].

The compounds present in the OSU-VLE Database III were classified in a similar manner as the UNIFAC functional-group classification approach [2]. The database is composed of compounds belonging to 31 chemical classes. Figure 1 illustrates the data distribution of the binary systems in the OSU database III based on chemical classes. The number of systems represented for each type of functional-group interaction is shown in the figure. Systems containing alcohol or alkane components are represented extensively in the database due to their abundant data.

\subsection{Interaction parameter regression methodology}

Regression analyses were conducted to optimize the adjustable parameter or parameters in the original NRTL, mNRTL2 and mNRTL1 models. The regression analyses were performed by applying the Gibbs equilibrium criteria for a closed system to the coexisting liquid and vapor phases, while subject to mass balance constraints. The split approach was employed in the phase equilibria calculations, as follows:

$$
\hat{\phi}_{i}^{V} P y_{i}=\gamma_{i} P_{i}^{\circ} \phi_{i}^{V} x_{i} \lambda_{i} ; \quad i=1, n
$$

where $\mathrm{n}$ is the number of components, the subscript $i$ represents a particular component, $\widehat{\emptyset}^{V}$ is the component fugacity coefficient in the vapor phase, $y$ is the vapor mole fraction, $\gamma$ is the component activity coefficient in the liquid phase, $P$ is the mixture pressure, $P^{\circ}$ is the purecomponent vapor pressure, $\emptyset^{V}$ is the pure-component fugacity coefficient in the vapor phase, $x$ is the liquid mole fraction and $\lambda$ is the Poynting factor. The VLE systems considered in this study were generally at low pressure; hence, the vapor-phase fugacity coefficients were 
assumed to be 1 . We have also investigated the quality of representation when equation-ofstate (EOS) models are used to calculate the vapor-phase fugacity coefficients. Our findings show there is no improvement on the overall representation error (results not shown). This result confirms that our assumption is reasonable.

The Poynting factor is expressed as follows:

$$
\lambda_{i}=\exp \left(\frac{v_{i}^{L}\left(P-P_{i}^{\circ}\right)}{R T}\right)
$$

where $v^{L}$ is the liquid molar volume and is determined using the Rackett equation [22].

The objective function, $O F$, used in the parameter regression analyses, was the weighted sums of squares of relative errors in pressure, K-values, infinite-dilution activity coefficients and weighted absolute sum of model parameters, as follows:

$$
\begin{aligned}
O F= & \sum_{i=1}^{n} w_{1}\left(\frac{P^{\text {Exp }}-P^{\text {Calc }}}{P^{\text {Exp }}}\right)_{i}^{2}+w_{2} \sum_{i=1}^{n}\left(\frac{K_{\text {values }}^{\text {Exp }}-K_{\text {values }}^{\text {Calc }}}{K_{\text {values }}^{\text {Exp }}}\right)_{i}^{2} \\
& +w_{3} \sum_{i=1}^{n}\left(\frac{\gamma_{\text {values }}^{\infty}-\gamma_{\text {values }}^{\infty \text { Calc }}}{\gamma_{\text {values }}^{\infty \text { Exp }}}\right)_{i}^{2}+w_{4}(\text { Par })
\end{aligned}
$$

where the weights were $w_{1}=1 ; w_{2}=1 / 15 ; w_{3}=1 / 10 ; w_{4}=2 E-6 ; n$ is the number of data points, Par is $\left|a_{12}\right|+\left|a_{21}\right|$ for the NRTL model and $\left|g_{12}\right|$ for the mNRTL1 model and the superscripts Exp and Calc refer to experimental and calculated values, respectively.

This objective function and associated weights were developed after evaluating the VLE property predictions employing various objective function formulations. Equation 14 was found to be the most suitable since the equation provided a balance of the model prediction errors for temperature, pressure, equilibrium constants, activity coefficient and vapor mole fraction and also reduced the correlation of the two model parameters ( $a_{12}$ and $a_{21}$ or $g_{12}$ and $R_{12}$ ) [23]. 


\subsection{Case studies}

Three regression case studies were conducted to investigate representation qualities of the original NRTL, mNRTL2 and mNRTL1 models. In all case studies, the ideal gas (IG) model was used to describe the gas phase behavior. The case studies are described below:

Case original NRTL: The original NRTL model was used to represent the activity coefficients by regressing $\mathrm{a}_{12}$ and $\mathrm{a}_{21}$.

Case mNRTL2: $\quad$ The $g_{12}$ and $R_{12}$ parameters in the modified NRTL model were regressed to represent the experimental data.

Case mNRTL1: $\quad$ The one-parameter modified NRTL model was evaluated by regressing $g_{12}$, with the second parameter set by Equation 11 .

The representation capabilities of the models were assessed for equilibrium properties such as pressure $(P)$, infinite-dilution activity coefficients $\left(\gamma^{\infty}\right)$, temperature $(T)$, component 1 vapor mole fraction $\left(y_{1}\right)$ and equilibrium K-value (average of $K_{1}$ and $K_{2}$ ). The regression was conducted by performing a bubble-point pressure calculation. After the regression analyses, the regressed parameters were used directly to calculate (a) $P, K_{1}$ and $K_{2}$ for known $T$ and $x_{1}$ and (b) $T$ for known $P$ and $x_{1}$.

\subsection{Behavior classification}

The degree of non-ideality depends on the particular types of molecular interactions encountered by the components of the system considered. Components with similar functional groups, polarity and sizes usually show nearly ideal behavior while components with a high degree of polarity difference exhibit highly non-ideal behavior. Although the types of molecules provide a general idea about mixture behaviors, they do not allow the precise determination of the degree of non-ideality [24]. The alternative is to employ a qualitative approach which relates model parameter values to the behavior of the systems. 
The ability to classify behaviors qualitatively of VLE systems is important in process design since this provides an easy method of determining the degree of non-ideality without the need of additional information. Once the type of behavior is identified, an appropriate thermodynamic model can be selected to determine properties of the systems.

Danner [24] presented a behavior classification approach based on the Margules model parameter $(A)$ for 104 VLE systems. In this approach, he presented the relationship of excess Gibbs energy to the $A$ parameter value. Danner classified systems with an $A$ value of between 0.6 and 0.6 to be nearly-ideal while a value greater than 0.6 or less than -0.6 were classified as highly non-ideal.

In this study, we have applied the same approach as Danner [24] to determine the $g_{12}$ values for ideal and non-ideal systems in the mNRTL1 model. Theoretically for ideal systems, the excess Gibbs energy is zero. In order to determine the cutoff point between ideal and non-ideal systems, we plotted $\mathrm{G}^{\mathrm{E}} / \mathrm{RT}$ as a function $\mathrm{x}_{1}$ for all the systems in our database. From the plots the maximum $\left|G^{\mathrm{E}} / \mathrm{RT}\right|$ values were determined and compared with the Margules $(\mathrm{A})$ and mNRTL1 $\left(g_{12}\right)$ model parameters.

Figure 2 shows the $\mathrm{G}^{\mathrm{E}} / \mathrm{RT}$ vs. $\mathrm{x}_{1}$ plots for six VLE binary systems. The systems were selected to demonstrate the change in behavior from nearly ideal to highly non-ideal systems. The degree of non-ideality increases as the maximum $\left|\mathrm{G}^{\mathrm{E}} / \mathrm{RT}\right|$ value increases. Table 1 shows the maximum $\left|G^{\mathrm{E}} / \mathrm{RT}\right|$ and $\gamma^{\infty}$ values for the selected six binary systems with regressed Margules $(A)$ and mNRTL1 $\left(g_{12}\right)$ model parameters. The table indicates for systems 2 and 5 the $|\mathrm{A}|$ parameter and maximum $\left|\mathrm{G}^{\mathrm{E}} / \mathrm{RT}\right|$ values are approximately 0.62 and 0.15 , respectively. In addition, the $\mathrm{\gamma}^{\infty}$ values for systems 2 and 5 are approximately 2 and 0.5, respectively. Based on the Danner [24] classification, these systems are classified as highly-non ideal. After examining all the systems in our database, the boundary for nearly ideal systems was found to occur at a maximum 
$\left|G^{\mathrm{E}} / \mathrm{RT}\right|$ value $<=0.15$. The relationship of maximum $\left|\mathrm{G}^{\mathrm{E}} / \mathrm{RT}\right|$ and the mNRTL1 $\left(g_{12}\right)$ model parameter value is discussed in the Results Section.

\section{Results and discussion}

The results of this study address five main concerns, which are (1) representation of equilibrium properties, (2) behavior classification, (3) cross-phase system predictions (the applicability of LLE regressed parameters to predict VLE properties and vice versa), (4) parameter temperature dependence and (5) multicomponent phase behavior predictions. The results of each focus is presented in the following sub sections.

\subsection{Regression of equilibrium properties}

The representation capabilities of the original NRTL, mNRTL2 and mNRTL1 models were assessed by using experimental $P, T, y_{1}, K$ and $\gamma^{\infty}$ values of 916 binary systems and $\gamma^{\infty}$ data of 137 binary systems. The regression errors from each model were analyzed by calculating the root-mean-squared error (RMSE), bias and \%AAD.

Table 2 provides the property representation errors for the original NRTL, mNRTL2 and mNRTL1 case studies. As shown in the table, the original NRTL model with regressed parameters provided overall \%AADs of $2.1,0.2,4.3,5.5$ and 8.7 for $P, T, y_{1}, K$ and $\gamma^{\infty}$, respectively. The mNRTL2 model provided overall \%AADs of $2.2,0.2,4.4,5.7$ and 10.2 for $P, T, y_{1}, K$ and $\gamma^{\infty}$, respectively. The results show the mNRTL2 provided comparable results to that of the original NRTL model. The one-parameter (mNRTL1) model resulted in overall \%AADs of $2.5,0.2,4.7,6.1$ and 13.3 for $P, T, y_{1}, K$ and $\gamma^{\infty}$, respectively. Compared to the mNRTL2 model, the mNRTL1 model provided good VLE property representation with a slight loss of precision. With only one parameter, the mNRTL1 was able to represent VLE properties including infinite-dilution activity coefficients $\left(\gamma^{\infty}\right)$, which has previously been a challenge for one-parameter models. 
Table 3 shows the property representation errors using the original NRTL, mNRTL2 and mNRTL1 models for binary VLE systems containing water. The property representations errors for water systems were slightly higher than the results found for the overall data. The mNRTL2 model resulted in comparable results with the original NRTL model for the water systems. The mNRTL1 model also provided reasonable precision in representing experimental data for water systems. These higher errors could be due to the high level of experimental uncertainty associated with water systems, and the inability of the models in representing such systems precisely. Further, the mole fraction of aqueous systems tend to be small which results in large percentage errors.

Figure 3 shows the distribution of pressure representation regression errors for the original NRTL, mNRTL2 and mNRTL1 models by functional-group interactions. As indicated by the error matrix, all three models have comparable representation capabilities for all type of interactions with the exception of the water systems. As expected, all models provided precise representations when the components in the system have the same functional groups (diagonal elements of the triangular matrix). The mNRTL1 model showed slightly higher errors for some of the interactions involving water. The results for water systems are inconclusive since the database lacks good representation of each type of interaction with water for a number of systems.

The representation quality of the proposed one-parameter model (mNRTL1) was compared with the Bruin-Prausnitz one-parameter model (NRVQR1) [9]. In the NRVQR1 model, liquid molar volume was determined using the Rackett equation [22], second virial coefficients were estimated using Tsonopoulos correlation [25] and, $\alpha_{12}$ and $\beta$ were fixed at values of 0.2 and 0.37, respectively. The NRVQR1 model with regressed $\Psi_{12}$ parameter resulted in overall \%AADs of $3.2,0.3,5.5,7.3$ and 20.9 for $\mathbb{P}_{w} \mathbb{T}_{w} \mathbb{Y}_{\mathbb{1}} \mathbb{K}$ and $\mathbb{P}^{\infty}$, respectively. This results show the NRVQR1 model provides relatively higher property representation errors compared to the 


\subsection{Behavior classification}

Table 4 shows the maximum $\left|\mathrm{G}^{\mathrm{E}} / \mathrm{RT}\right|$ range of values that were used to identify the degree of non-ideality in the VLE systems. Systems with a maximum $\left|\mathrm{G}^{\mathrm{E}} / \mathrm{RT}\right|$ value $<=0.15$ are classified as nearly-ideal, while maximum $\left|\mathrm{G}^{\mathrm{E}} / \mathrm{RT}\right|$ values $>0.15$ are classified as highly non-ideal systems.

Figure 4 shows the distribution of $g_{12}$ values based on maximum $\left|\mathrm{G}^{\mathrm{E}} / \mathrm{RT}\right|$ values for 913 systems. The figure shows the nearly-ideal system $g_{12}$ range (approximately between -170 and 220) and the highly non-ideal system ranges. As indicated in the figure, the highly non-ideal system range overlaps the nearly-ideal system range on both the left and right sides. To avoid misclassifying systems in the overlapping region, we considered only correctly classified systems in Figure 5, which eliminated the overlap shown in Figure 4.

Table 5 provides the range of the $g_{12}$ parameter for nearly-ideal and highly non-ideal system classes excluding those systems that are in the overlapping region. The result shows the $g_{12}$ range of the nearly-ideal systems is approximately between -100 and 100 while highly non-ideal systems are $>220$ and $<-180$. The systems in the overlapping regions could be considered as non-ideal systems due to the fact that they cannot be classified as nearly-ideal or highly nonideal. The range of $g_{12}$ for non-ideal systems are between -180 and -100 and 100 and 220 . The classification results confirm that when the interaction energy value increases the degree of the non-ideality also increases.

Table 6 shows the pressure property representation errors using the original NRTL, mNRTL2 and mNRTL1 models for nearly-ideal, non-ideal and highly non-ideal systems. As expected, the representation quality decreases as degree of non-ideality increases. The result also shows the 
three models have comparable representation capability for nearly-ideal systems. For non-ideal and highly non-ideal systems, the original NRTL model provided slightly better representation of pressure compared to the modified NRTL models.

\subsection{Cross-phase property predictions}

The representation capabilities of the original NRTL and mNRTL2 models were evaluated using VLE and LLE experimental data. In this study, twenty systems with both binary VLE and LLE experimental data were gathered from the VLE and LLE DECHEMA databases [10, 14]. Regression analyses were carried out for VLE, LLE and VLE-LLE (LLE and VLE data combined) systems. The original NRTL and mNRTL2 model parameters found in the regression analyses were different for the VLE and LLE systems with the same components. We investigated the source effect of model parameters (from VLE or LLE or VLE-LLE regressions) on the property predictions of different phases.

Tables 7 and 8 show the regression results of the 20 VLE, LLE and VLE-LLE binary systems using the original NRTL and mNRTL2 models. The results show the two models have comparable representation capability for correlating experimental mole fractions. The \%AADs on liquid mole fraction were approximately 18 and 20 for LLE and VLE systems, respectively. The error for the combined VLE-LLE data increased slightly to $26 \%$ for both models.

The robustness of the two models was investigated by predicting equilibrium properties of VLE, LLE and VLE-LLE systems using NRTL parameters regressed only using LLE, VLE or VLE-LLE data. Tables 7 and 8 show the prediction of $x_{1}$ for VLE and $x_{1}$ in Phase 1 for LLE and VLE-LLE data using NRTL parameters regressed only from LLE, VLE or VLE-LLE data. Using both models, the results show parameters from the VLE-LLE regression provided the lowest errors when used for VLE and LLE systems. In both models, the LLE parameters resulted in relatively better VLE and VLE-LLE property prediction compared to the VLE parameters when used for LLE and VLE-LLE systems. 
This study revealed the lack of robustness of the NRTL model in handling both VLE and LLE properties with the same regressed parameters. This could be due to the strong temperature dependence of the model parameters, especially for the LLE systems. Improved accounting for the temperature dependence of LLE systems may be attained incorporating equation-of-state interaction concepts within the modified NRTL model.

\subsection{Temperature dependence of the MNRTL2 model $g_{12}$ parameter}

The effect of temperature on the $g_{12}$ parameter of the mNRTL2 model was examined. Six LLE and VLE systems listed in Table 9 were collected from the DECHEMA VLE [10] and DECHEMA LLE databases [14]. Regression analyses were carried out to determine the optimum value of $g_{12}$ and $R_{12}$ parameters for LLE and VLE systems. In the regression analysis, the $\mathrm{R}_{12}$ values were fixed at the LLE regressed value while $g_{12}$ is regressed temperature by temperature for both the LLE and VLE systems.

Figures $6 \mathrm{a}$ and $6 \mathrm{~b}$ show the $\mathrm{g}_{12}$ temperature by temperature regression results of the six VLE and LLE systems. The error bars indicate the range of $g_{12}$ values that correspond to a $\pm 25 \%$ increase in the property prediction errors. The dotted lines are drawn to clearly indicate $g_{12}$ values that belong to a same component VLE and LLE mixture. Systems 1, 2, 3 and 4 show the value of $g_{12}$ increases as temperature increases, which indicates that VLE systems tend to have higher $g_{12}$ values than LLE systems. The two exceptions are Systems 5 and 6 where the results show an inverse relationship of $g_{12}$ and temperature. In general, the $g_{12}$ parameter has a linear type of relationship with temperature for the LLE and VLE systems.

\subsection{Multicomponent phase behavior predictions}

The prediction capabilities of the original NRTL, mNRTL2 and mNRTL1 models were evaluated for multicomponent systems. The objectives of this study is to assess the representation 
capability of the three models for ternary VLE property predictions using interaction parameters obtained from regression of binary VLE experimental data. To accomplish this, we assembled a database of 57 ternary VLE systems encompassing a variety of molecular species. Regressed binary model parameters were used to predict the phase equilibrium properties of the ternary systems.

Table 10 shows the prediction of ternary properties using the original NRTL, mNRTL2 and mNRTL1 models. The original NRTL equation resulted in \%AADs of approximately 3, 0.3 and 9 for pressure, temperature and K-value predictions, respectively. Compared to the original NRTL, the mNRTL2 and mNRTL1 model resulted in slightly higher \%AADs, which is attributed to four systems that exhibited larger deviations than the common trend. When these four systems were removed, all three models (NRTL, mNRTL1, and mNRTL2) give comparable results for ternary systems. It should be noted that while some systems may yield worse deviations for the modified models, other systems show worse results for the original NRTL. This is illustrated well by the following two ternary mixtures. For methanol-benzene-cyclohexane, toluenecyclohexane-ethanol and ethanol-benzene-cyclohexane systems, the mNRTL2 and mNRTL1 models resulted in about 2 to 4 times the pressure errors found using the original NRTL model. Whereas, for ternary systems such as, methanol-vinyl acetate-cyclohexane, acetone-methanolbenzene and ethanol-acetone-benzene, the original NRTL model provided 2 to 3 times the pressure errors compared to the mNRTL2 and mNRTL1 model results. In all cases, however, the averaged errors are within 1.5 times the overall errors found from binary system regression analyses. The results indicate all three models could be extended to multicomponent phase behavior predictions with only a slight loss of accuracy.

\section{Conclusion}

In this study, we proposed a modification to the widely used NRTL activity coefficient model which addresses the limitation of the original model. The representation capabilities of the 
models were assessed with 916 VLE and 20 binary LLE systems. The regression results indicate the newly proposed model provides comparable results with the original NRTL model.

The study provided a generalization for the interaction ratio in the newly proposed model using pure-component properties. This reduces the model to only one energy interaction parameter and eliminates the correlation between parameters. Compared to the original NRTL model, the one-parameter model provided VLE equilibrium property representations with a slight loss of precision. A study is underway to further generalize the model by relating the energy interaction parameter to the structures of molecules in the binary systems.

Model parameters for VLE and LLE systems are different for both the original and modified models. Further, the model parameters in both models show strong temperature dependence for the LLE systems. This suggests a possible improvement in the temperature dependence of the NRTL model.

\section{Acknowledgements}

This material is based on work partially supported by the U.S. Department of Energy, DOE/EPSCOR (Grant DE-SC0004600). 


\section{References}

1. Renon, H. and J.M. Prausnitz, Local compositions in thermodynamic excess functions for liquid mixtures. AIChE Journal, 1968. 14(1): p. 135-144.

2. Gmehling, J., J. Li, and M. Schiller, A modified UNIFAC model. 2. Present parameter matrix and results for different thermodynamic properties. Industrial \& Engineering Chemistry Research, 1993. 32(1): p. 178-193.

3. Abrams, D.S. and J.M. Prausnitz, Statistical thermodynamics of liquid mixtures: A new expression for the excess Gibbs energy of partly or completely miscible systems. AIChE Journal, 1975. 21(1): p. 116-128.

4. Skjold-Jorgensen, S., B. Kolbe, J. Gmehling, and P. Rasmussen, Vapor-liquid equilibria by UNIFAC group contribution. Revision and extension. Industrial \& Engineering Chemistry Process Design and Development, 1979. 18(4): p. 714-722.

5. Fischer, K. and J. Gmehling, Further development, status and results of the PSRK method for the prediction of vapor-liquid equilibria and gas solubilities. Fluid Phase Equilibria, 1996. 121(1-2): p. 185-206.

6. Prausnitz, J.M., R.N. Lichtenthaler, and E.G.d. Azevedo, Molecular thermodynamics of fluid-phase equilibria. 3rd ed. 1998: Prentice-Hall.

7. Wilson, G.M., Vapor-liquid equilibrium. XI. A new expression for the excess free energy of mixing. Journal of the American Chemical Society, 1964. 86(2): p. 127-130.

8. Gmehling, J., D. Tiegs, and U. Knipp, A comparison of the predictive capability of different group contribution methods. Fluid Phase Equilibria, 1990. 54: p. 147-165.

9. Bruin, S. and J.M. Prausnitz, One-Parameter Equation for Excess Gibbs Energy of Strongly Nonideal Liquid Mixtures. Industrial \& Engineering Chemistry Process Design and Development, 1971. 10(4): p. 562-572.

10. Gmehling, J., U. Onken, and W. Arlt, Vapor-liquid equilibrium data collection. Chemistry Data Series. Vol. I, Parts 1-8. 1977 - 2001: DECHEMA, Frankfurt, Germany. 
11. Vetere, A., An improved method to predict VLE equilibria of subcritical mixtures. Fluid Phase Equilibria, 1996. 124(1-2): p. 15-29.

12. Vetere, A., Prediction of vapor-liquid equilibria of aqueous systems in the subcritical range by using the NRTL equation. Fluid Phase Equilibria, 1994. 99(0): p. 63-74.

13. Vetere, A., Prediction of vapor-liquid equilibria of non-aqueous systems in the subcritical range by using the NRTL equation. Fluid Phase Equilibria, 1993. 91(2): p. 265-280.

14. Arlt, W., M.E.A. Macedo, P. Rasmussen, and J.M. Sorensen, Liquid-liquid equilibrium data collection. Chemistry Data Series. Vol. V, Parts 1-4. 1979 - 1987: DECHEMA, Frankfurt, Germany.

15. NIST-TDE, NIST Standard Reference Database 103b ThermoData Engine. 2012.

16. DIPPR Project 801, Physical and Thermodynamic Properties of Pure Chemicals. 2011.

17. Scatchard, G., S.E. Wood, and J.M. Mochel, Vapor-liquid equilibrium. VII. Carbon tetrachloride-methanol mixtures 1. Journal of the American Chemical Society, 1946. 68(10): p. 1960-1963.

18. Vetere, A., A modified Heil-Prausnitz equation for excess gibbs energy. The Canadian Journal of Chemical Engineering, 1977. 55(1): p. 70-77.

19. Hansen, H.K., P. Rasmussen, A. Fredenslund, M. Schiller, and J. Gmehling, Vaporliquid equilibria by UNIFAC group contribution. 5. Revision and extension. Industrial \& Engineering Chemistry Research, 1991. 30(10): p. 2352-2355.

20. Gao, W., R.L. Robinson Jr, and K.A.M. Gasem, Alternate equation of state combining rules and interaction parameter generalizations for asymmetric mixtures. Fluid Phase Equilibria, 2003. 213(1-2): p. 19-37.

21. Ravindranath, D., B.J. Neely, R.L. Robinson Jr., and K.A.M. Gasem, QSPR generalization of activity coefficient models for predicting vapor-liquid equilibrium behavior. Fluid Phase Equilibria, 2007. 257(1): p. 53-62.

22. Rackett, H.G., Equation of state for saturated liquids. Journal of Chemical and Engineering Data, 1970. 15(4): p. 514-517. 
23. Tassios, D., The number of roots in the NRTL and LEMF equations and the effect on their performance. Industrial \& Engineering Chemistry Process Design and Development, 1979. 18(1): p. 182-186.

24. Danner, R.P. and M.A. Gess, A data base standard for the evaluation of vapor-liquidequilibrium models. Fluid Phase Equilibria, 1990. 56(0): p. 285-301.

25. Tsonopoulos, C., An empirical correlation of second virial coefficients. AIChE Journal, 1974. 20(2): p. 263-272. 
1. Maximum $\left|\mathrm{G}^{\mathrm{E}} / \mathrm{RT}\right|$ and $\mathrm{y}^{\infty}$ properties for five VLE binary systems with their regressed Margules (A) and mNRTL1 $\left(g_{12}\right)$ parameters

2. VLE property representation capability of the original NRTL, mNRTL2 and mNRTL1 models

3. VLE property representation capability of the original NRTL, mNRTL2 and mNRTL1 models for water systems

4. Classification of binary systems based on $\max \left|\mathrm{G}^{\mathrm{E}} / \mathrm{RT}\right|$

5. Range of parameters excluding systems that are in the overlapping region

6. Regression results of the original NRTL, mNRTL2 and mNRTL1 by type of behavior

7. Regression results of 20 VLE and LLE systems using the original NRTL model

8. Regression results of 20 VLE and LLE systems using the mNRTL2 model

9. LLE and VLE systems used for the temperature dependence study of the $g_{12}$ parameter

10. Prediction results of 57 ternary VLE systems using the original NRTL, mNRTL2 and mNRTL1 models 
Table 1. Maximum $\left|G^{E} / R T\right|$ and $y^{\infty}$ properties for six VLE binary systems with their regressed Margules (A) and mNRTL1 $\left(g_{12}\right)$ parameters

\begin{tabular}{|c|c|c|c|c|c|c|c|c|}
\hline \multirow[b]{2}{*}{ Sys } & \multirow[b]{2}{*}{ Type } & \multirow[b]{2}{*}{ Comp 1} & \multirow[b]{2}{*}{ Comp 2} & \multirow[b]{2}{*}{ A } & \multirow[b]{2}{*}{$g_{12}$} & \multicolumn{3}{|c|}{ Original NRTL } \\
\hline & & & & & & $\underset{\left|G^{E} / R T\right|}{\operatorname{Max}}$ & $Y^{\infty}$ & $\mathrm{Y}^{\infty}{ }_{2}$ \\
\hline 1 & Positive & n-octane & ethylbenzene & 0.19 & 66 & 0.05 & 1.2 & 1.2 \\
\hline 2 & Excess & 1,2-dichloroethane & tetrachloroethylene & 0.62 & 236 & 0.16 & 1.9 & 1.8 \\
\hline 3 & Gibbs & ethyl tertiary butyl ether & ethanol & 1.43 & 547 & 0.37 & 3.5 & 5.9 \\
\hline 4 & Negative & methanol & pyridine & -0.12 & -37 & -0.04 & 0.9 & 0.8 \\
\hline 5 & Excess & hexafluorobenzene & p-xylene & -0.62 & -182 & -0.16 & 0.6 & 0.5 \\
\hline 6 & Gibbs & butylamine & 1-propanol & -1.25 & -338 & -0.31 & 0.2 & 0.3 \\
\hline
\end{tabular}

Table 2. VLE property representation capability of the original NRTL, mNRTL2 and mNRTL1 models

\begin{tabular}{|c|c|c|c|c|c|c|c|}
\hline Model (L) & Parameters & Property & $\begin{array}{l}\text { No. } \\
\text { sys }\end{array}$ & No. pts & RMSE & Bias & $\% A A D$ \\
\hline \multirow{5}{*}{$\begin{array}{c}\text { Original } \\
\text { NRTL }\end{array}$} & \multirow{5}{*}{$a_{12} \& a_{21}$} & $\mathrm{P}$ (bar) & 916 & 33841 & 0.15 & 0.00 & 2.1 \\
\hline & & $\mathrm{T}(\mathrm{K})$ & 916 & 33841 & 1.35 & 0.10 & 0.2 \\
\hline & & $\mathrm{y}_{1}$ & 675 & 18199 & 0.03 & 0.00 & 4.3 \\
\hline & & K-value & 675 & 18199 & 5.09 & -0.31 & 5.5 \\
\hline & & $y^{\infty}$ & 137 & 549 & 3.54 & -0.21 & 8.7 \\
\hline \multirow{5}{*}{ mNRTL2 } & \multirow{5}{*}{$g_{12} \& R_{12}$} & $\mathrm{P}$ (bar) & 916 & 33844 & 0.17 & 0.00 & 2.2 \\
\hline & & $\mathrm{T}(\mathrm{K})$ & 916 & 33844 & 1.42 & 0.11 & 0.2 \\
\hline & & $\mathrm{y}_{1}$ & 675 & 18199 & 0.03 & 0.00 & 4.4 \\
\hline & & K-value & 675 & 18199 & 4.84 & -0.28 & 5.7 \\
\hline & & $\gamma^{\infty}$ & 137 & 549 & 4.70 & -0.34 & 10.2 \\
\hline \multirow{5}{*}{ mNRTL1 } & \multirow{5}{*}{$g_{12}$} & $\mathrm{P}$ (bar) & 916 & 33845 & 0.24 & -0.01 & 2.5 \\
\hline & & $\mathrm{T}(\mathrm{K})$ & 916 & 33845 & 1.67 & 0.16 & 0.2 \\
\hline & & $y_{1}$ & 675 & 18199 & 0.03 & 0.00 & 4.7 \\
\hline & & K-value & 675 & 18199 & 5.41 & -0.21 & 6.1 \\
\hline & & $\gamma^{\infty}$ & 137 & 549 & 6.46 & -0.77 & 13.3 \\
\hline
\end{tabular}


Table 3. VLE property representation capability of the original NRTL, mNRTL2 and mNRTL1 models for water systems

\begin{tabular}{|c|c|c|c|c|c|c|c|}
\hline Model & Parameters & Property & No. sys & No. pts & RMSE & Bias & $\% A A D$ \\
\hline \multirow{4}{*}{$\begin{array}{c}\text { Original } \\
\text { NRTL }\end{array}$} & \multirow{4}{*}{$a_{12} \& a_{21}$} & $\mathrm{P}$ (bar) & 55 & 4344 & 0.40 & -0.02 & 4.8 \\
\hline & & $\mathrm{T}(\mathrm{K})$ & 55 & 4344 & 2.47 & 0.41 & 0.4 \\
\hline & & $y_{1}$ & 47 & 2313 & 0.06 & -0.01 & 10.6 \\
\hline & & K-value & 47 & 2313 & 17.40 & -3.98 & 11.6 \\
\hline \multirow{4}{*}{ mNRTL2 } & \multirow{4}{*}{$g_{12} \& R_{12}$} & $\mathrm{P}$ (bar) & 55 & 4344 & 0.35 & -0.02 & 5.7 \\
\hline & & $\mathrm{T}(\mathrm{K})$ & 55 & 4344 & 2.72 & 0.51 & 0.5 \\
\hline & & $\mathrm{y}_{1}$ & 47 & 2313 & 0.07 & -0.01 & 11.7 \\
\hline & & K-value & 47 & 2313 & 16.29 & -3.57 & 13.2 \\
\hline \multirow{4}{*}{ mNRTL1 } & \multirow{4}{*}{$g_{12}$} & $\mathrm{P}$ (bar) & 55 & 4344 & 0.70 & -0.10 & 7.2 \\
\hline & & $\mathrm{T}(\mathrm{K})$ & 55 & 4344 & 3.99 & 1.11 & 0.6 \\
\hline & & $\mathrm{y}_{1}$ & 47 & 2313 & 0.08 & 0.00 & 13.2 \\
\hline & & K-value & 47 & 2313 & 18.44 & -2.41 & 15.6 \\
\hline
\end{tabular}

Table 4. Classification of binary systems based on $\operatorname{Max}\left|G^{\mathrm{E}} / \mathrm{RT}\right|$

\begin{tabular}{ccc}
\hline No & Range & Type \\
\hline 1 & $\operatorname{Max}\left|\mathrm{G}^{\mathrm{E}} / \mathrm{RT}\right|<=0.15$ & Nearly-ideal \\
2 & $\operatorname{Max}\left|\mathrm{G}^{\mathrm{E}} / \mathrm{RT}\right|>0.15$ & Highly non-ideal \\
\hline
\end{tabular}

Table 5. Range of parameters excluding systems that are in the overlapping region

\begin{tabular}{cccccc}
\hline No & Type & Max $\mathrm{G}^{\mathrm{E}} / \mathrm{RT}$ Range & $\boldsymbol{g}_{12}$ Range & $\begin{array}{c}\text { Average } \\
\text { po }\end{array}$ & No. sys \\
\hline 1 & Nearly-ideal & $\operatorname{Max}\left|\mathrm{G}^{\mathrm{E}} / \mathrm{RT}\right|<=0.15$ & $\left|g_{12}\right|<=100$ & 1.1 & 401 \\
& & & $-180<g_{12}<-100$ & 2.9 & 167 \\
2 & Non-ideal & $\operatorname{Max}\left|\mathrm{G}^{\mathrm{E}} / \mathrm{RT}\right| \approx 0.15$ & $\& 100<g_{12}<-220$ & & \\
& & & $g_{12}<=-180$ & 0.3 & 21 \\
3 & Highly non-ideal & $\operatorname{Max}\left|\mathrm{G}^{\mathrm{E}} / \mathrm{RT}\right|>0.15$ & $g_{12}>=220$ & 12 & 324 \\
\hline & Highly non-ideal & $\operatorname{Max}\left|\mathrm{G}^{\mathrm{E}} / \mathrm{RT}\right|<-0.15$ & & &
\end{tabular}


Table 6. Regression results of the original NRTL, mNRTL2 and mNRTL1 by type of behavior

\begin{tabular}{ccccc}
\hline \multirow{2}{*}{ Type } & No. sys & \multicolumn{3}{c}{ \%AAD on pressure } \\
\cline { 3 - 5 } & & Original NRTL & mNRTL2 & mNRTL1 \\
\hline Nearly-ideal & 401 & 1.6 & 1.6 & 1.6 \\
Non-ideal & 167 & 1.9 & 2.3 & 2.4 \\
Highly non-ideal & 345 & 2.7 & 2.9 & 3.6 \\
\hline
\end{tabular}

Table 7. Regression results of 20 VLE and LLE systems using the original NRTL model

\begin{tabular}{|c|c|c|c|c|c|c|}
\hline \multirow[b]{2}{*}{ Type of data } & \multirow[b]{2}{*}{$\begin{array}{l}\text { No. } \\
\text { sys }\end{array}$} & \multirow[b]{2}{*}{$\begin{array}{l}\text { No. } \\
\text { pts }\end{array}$} & \multicolumn{4}{|c|}{$\% A A D$ on for $x_{1}$ for VLE and $x_{1}$ in Phase 1 for LLE } \\
\hline & & & $\begin{array}{c}\text { Original } \\
\text { NRTL } \\
\text { Regression }\end{array}$ & $\begin{array}{l}\text { Parameters } \\
\text { from LLE } \\
\text { Regression }\end{array}$ & $\begin{array}{l}\text { Parameters } \\
\text { from VLE } \\
\text { Regression }\end{array}$ & $\begin{array}{c}\text { Parameters } \\
\text { from VLE- } \\
\text { LLE }\end{array}$ \\
\hline LLE & 20 & 108 & 18.0 & 18.0 & 61.8 & 33.2 \\
\hline VLE & 20 & 1231 & 19.9 & 34.0 & 19.9 & 25.9 \\
\hline VLE-LLE & 20 & 1340 & 25.0 & 31.3 & 37.4 & 25.0 \\
\hline
\end{tabular}

Table 8. Regression results of 20 VLE and LLE systems using the mNRTL2 model

\begin{tabular}{|c|c|c|c|c|c|c|}
\hline \multirow[b]{2}{*}{ Type of data } & \multirow[b]{2}{*}{$\begin{array}{l}\text { No. } \\
\text { sys }\end{array}$} & \multirow[b]{2}{*}{$\begin{array}{l}\text { No. } \\
\text { pts }\end{array}$} & \multicolumn{4}{|c|}{$\% A A D$ on $x_{1}$ for VLE and $x_{1}$ in Phase 1 for LLE } \\
\hline & & & $\begin{array}{c}\text { mNRTL2 } \\
\text { Regression }\end{array}$ & $\begin{array}{l}\text { Parameters } \\
\text { from LLE } \\
\text { Regression }\end{array}$ & $\begin{array}{l}\text { Parameters } \\
\text { from VLE } \\
\text { Regression }\end{array}$ & $\begin{array}{c}\text { Parameters } \\
\text { from VLE- } \\
\text { LLE }\end{array}$ \\
\hline LLE & 20 & 108 & 17.8 & 17.8 & 64.1 & 32.0 \\
\hline VLE & 20 & 1231 & 20.1 & 34.9 & 20.1 & 27.8 \\
\hline VLE-LLE & 20 & 1340 & 26.6 & 32.0 & 38.3 & 26.6 \\
\hline
\end{tabular}


Table 9. LLE and VLE systems used for the temperature dependence study of the $g_{12}$ parameter

\begin{tabular}{cc}
\hline No & System \\
\hline 1 & methanol + hexane \\
2 & diethyl ether + water \\
3 & diisopropyl ether + water \\
\hline
\end{tabular}

\begin{tabular}{cc}
\hline No & System \\
\hline 4 & methanol + cyclohexanol \\
5 & acetonitrile + Water \\
6 & nitromethane + cyclohexane \\
\hline
\end{tabular}

Table 10. Prediction results of 57 ternary VLE systems using the original NRTL, mNRTL2 and mNRTL1 models

\begin{tabular}{|c|c|c|c|c|c|c|c|}
\hline Study & Parameters & No. sys & Property & No. pts & RMSE & Bias & $\% A A D$ \\
\hline \multirow{4}{*}{$\begin{array}{c}\text { Original } \\
\text { NRTL }\end{array}$} & \multirow{4}{*}{$a_{12} \& a_{21}$} & \multirow{4}{*}{57} & $\mathrm{P}$ (bar) & 2212 & 0.05 & 0.00 & 3.0 \\
\hline & & & $\mathrm{T}(\mathrm{K})$ & 2212 & 1.57 & 0.16 & 0.3 \\
\hline & & & $y_{1}$ & 1890 & 0.04 & 0.00 & 8.7 \\
\hline & & & K-values & 1890 & 0.43 & 0.01 & 8.3 \\
\hline \multirow{4}{*}{ mNRTL2 } & \multirow{4}{*}{$g_{12} \& R_{21}$} & \multirow{4}{*}{57} & $\mathrm{P}$ (bar) & 2212 & 0.05 & 0.01 & 3.7 \\
\hline & & & $\mathrm{T}(\mathrm{K})$ & 2212 & 1.71 & -0.29 & 0.3 \\
\hline & & & $\mathrm{y}_{1}$ & 1890 & 0.04 & 0.00 & 9.6 \\
\hline & & & K-values & 1890 & 0.37 & 0.02 & 9.1 \\
\hline \multirow{4}{*}{ mNRTL1 } & \multirow{4}{*}{$g_{12}$} & \multirow{4}{*}{57} & $\mathrm{P}$ (bar) & 2212 & 0.05 & 0.01 & 3.8 \\
\hline & & & $\mathrm{T}(\mathrm{K})$ & 2212 & 1.71 & -0.31 & 0.3 \\
\hline & & & $\mathrm{y}_{1}$ & 1890 & 0.04 & 0.00 & 9.9 \\
\hline & & & K-values & 1890 & 0.38 & 0.02 & 9.4 \\
\hline
\end{tabular}


List of Figures

1. Database matrix of the compounds in the OSU-VLE database III

2. Excess Gibbs energy for five VLE binary systems in the OSU-VLE-III database

3. Pressure representation of the regressed NRTL, mNRTL1 and mNRTL2 models by type of interaction

4. Distribution of $g_{12}$ based on maximum $\left|\mathrm{G}^{\mathrm{E}} / \mathrm{RT}\right|$ values in the OSU-VLE-III database

5. Distribution of $g_{12}$ based on maximum $\left|\mathrm{G}^{\mathrm{E}} / \mathrm{RT}\right|$ values excluding overlapping region systems

6. Variation of $g_{12}$ with temperature for VLE and LLE systems where $\mathrm{R}_{12}$ is fixed as the LLE regressed value (a) systems 1-3 and (b) systems 4-6 


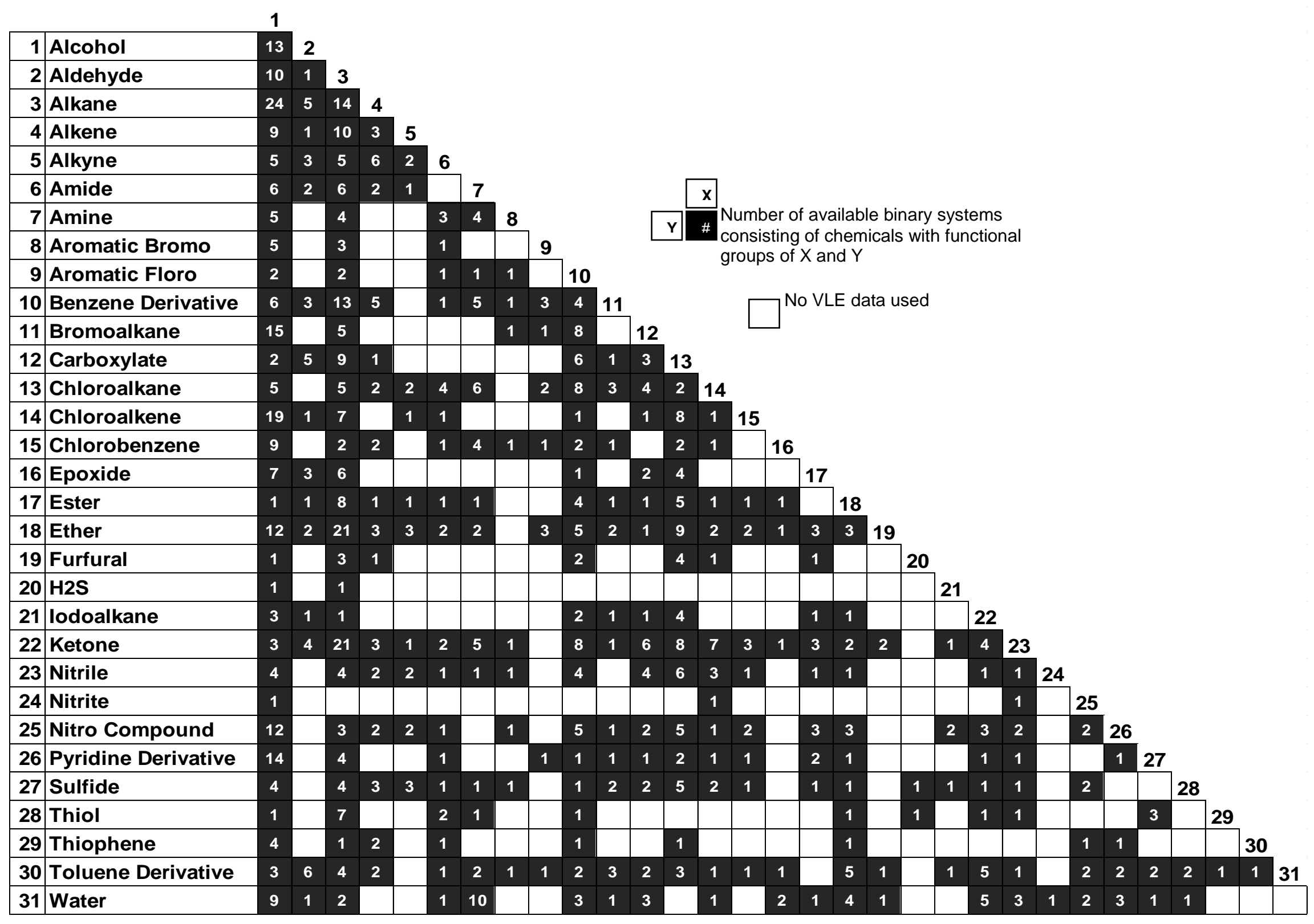

Figure 1. Database matrix of the compounds in the OSU-VLE database III 


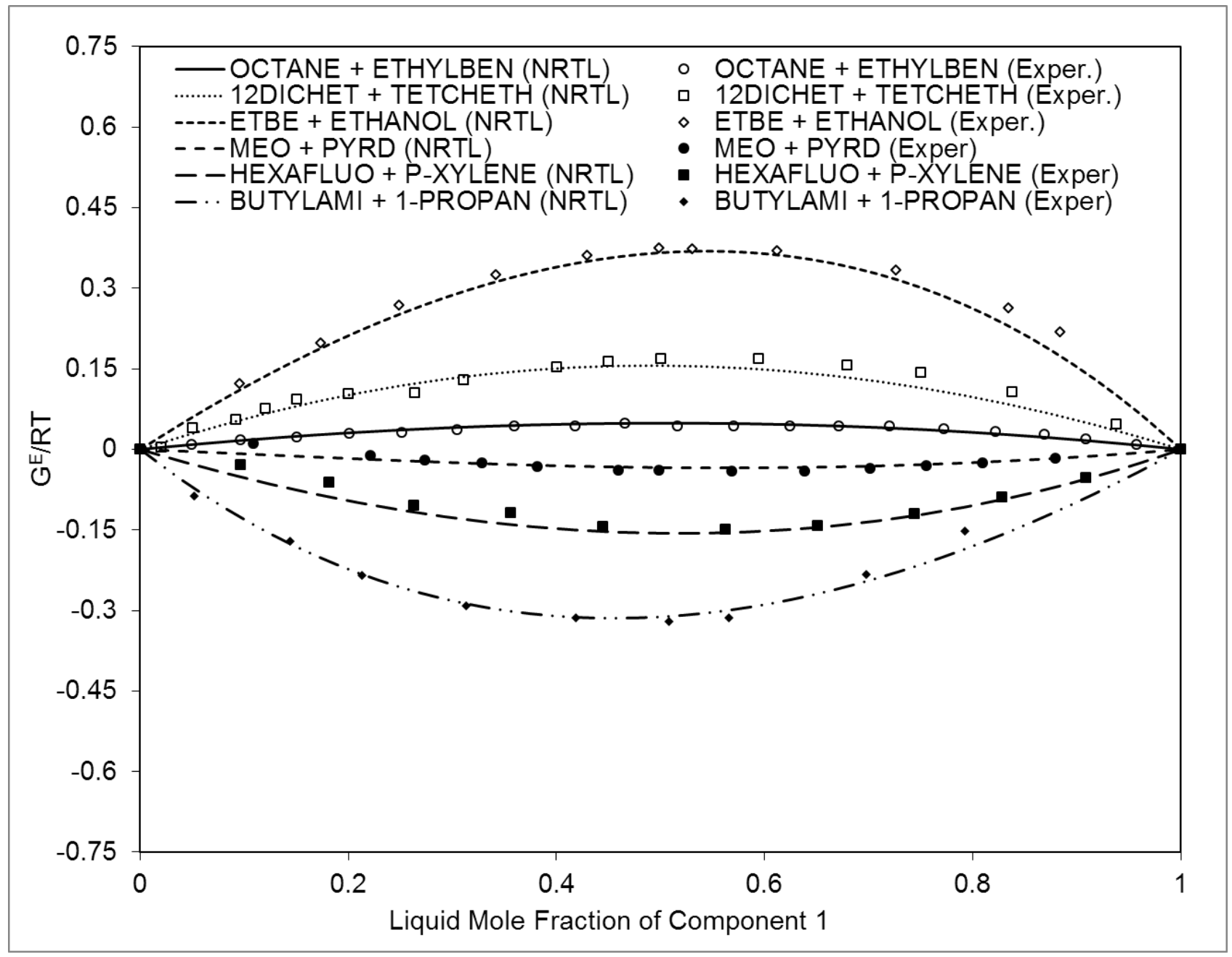

Figure 2. Excess Gibbs energy for six VLE binary systems in the OSU-VLE-III database 


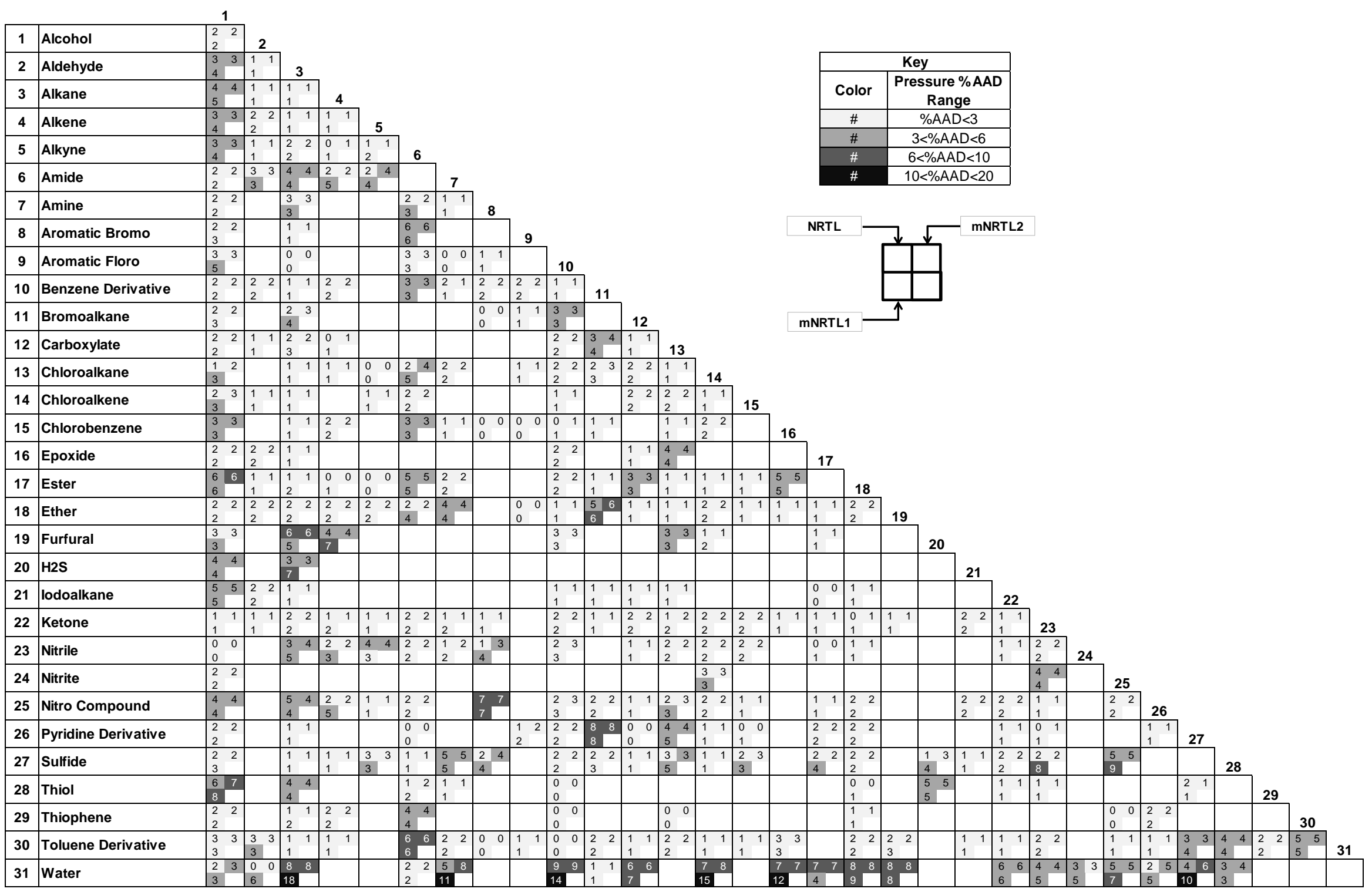

Figure 3. Pressure representation of the original NRTL, mNRTL1 and mNRTL2 models by type of interaction 


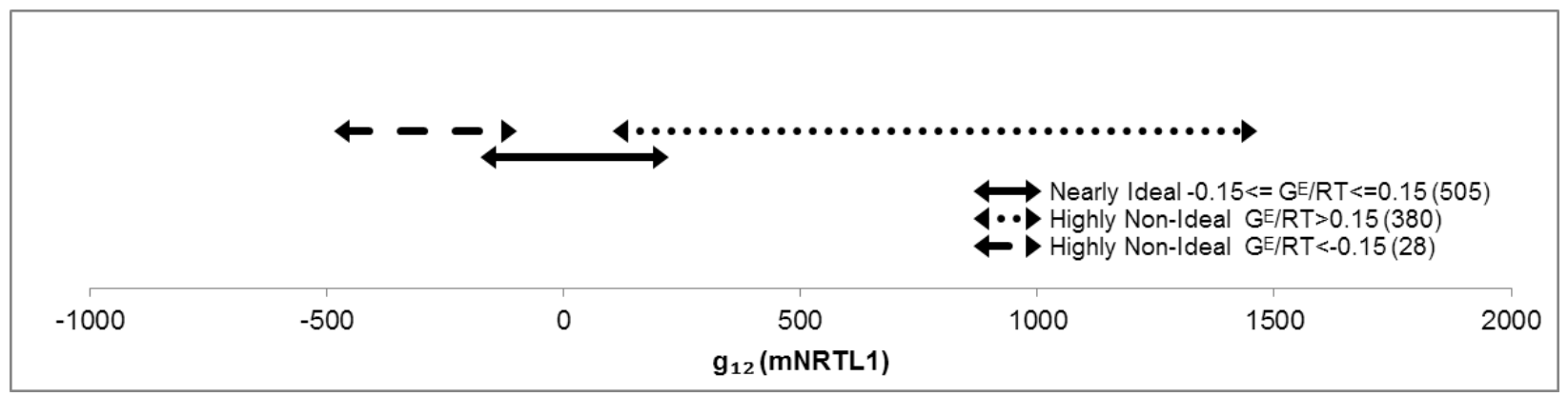

Figure 4. Distribution of $g_{12}$ based on maximum $\left|\mathrm{G}^{\mathrm{E}} / \mathrm{RT}\right|$ values in the OSU-VLE-III database

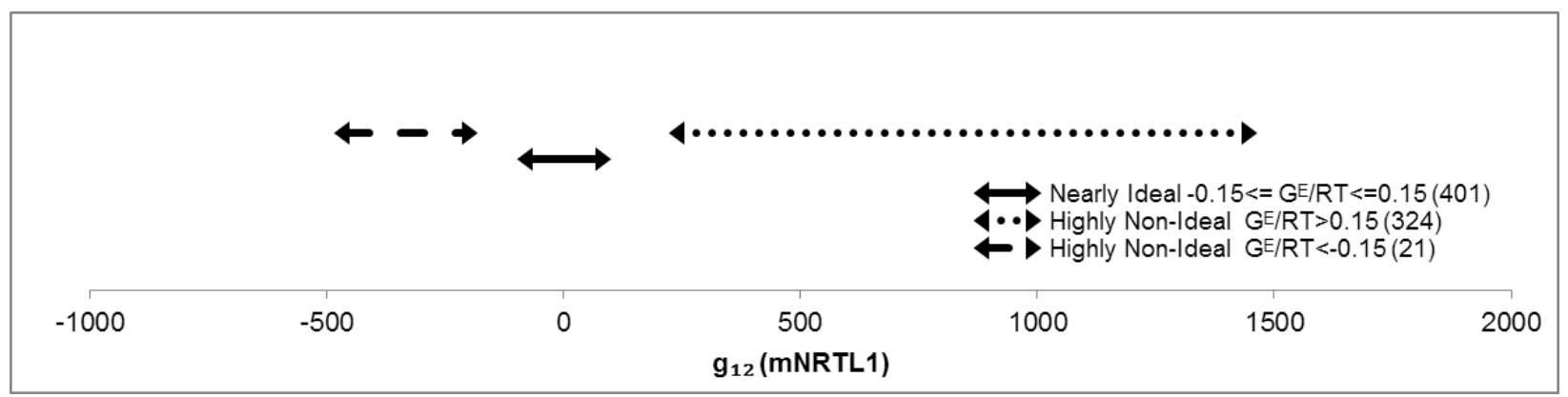

Figure 5. Distribution of $g_{12}$ based on maximum $\left|\mathrm{G}^{\mathrm{E}} / \mathrm{RT}\right|$ values excluding overlapping region systems 

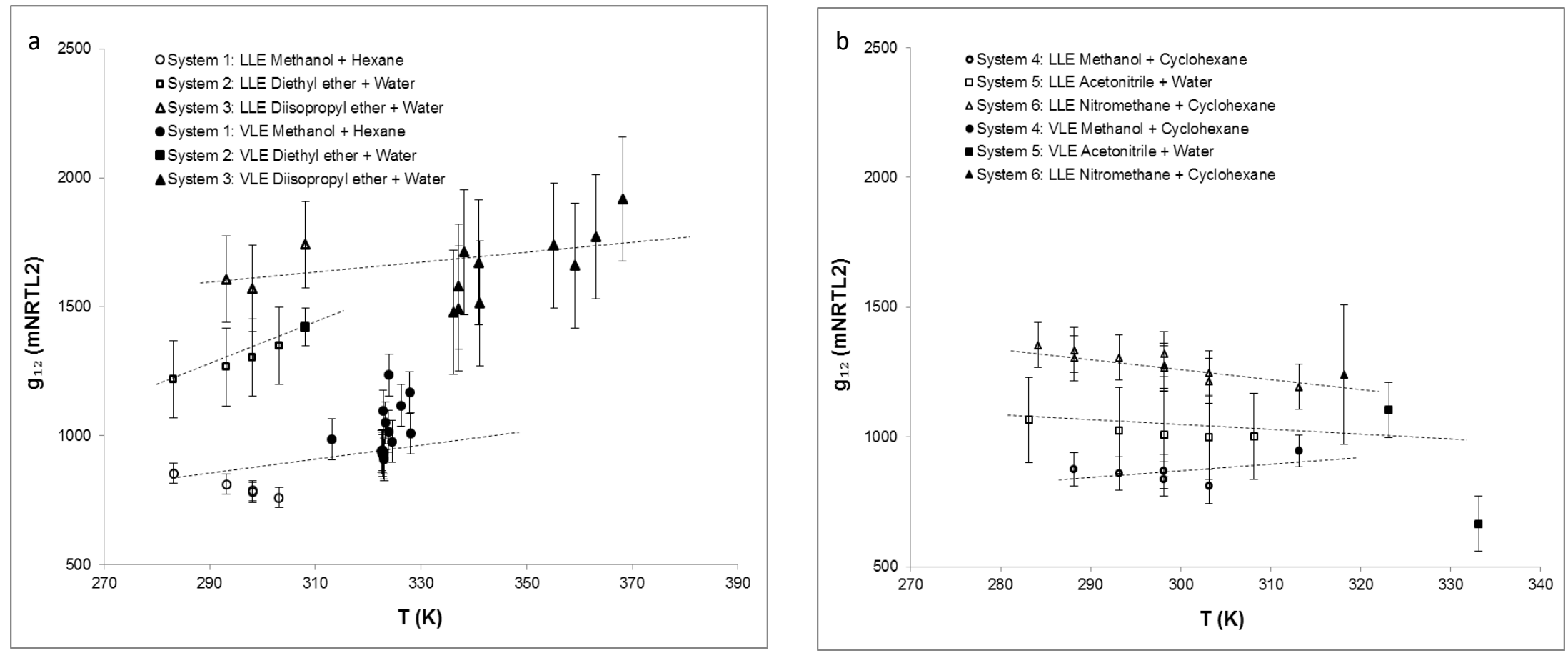

Figure 6. Variation of $g_{12}$ with temperature for VLE and LLE systems where $R_{12}$ is fixed as the LLE regressed value (a) systems $1-3$ and (b) systems 4-6 